\title{
De la Théorie à la Pratique de la Décision médicale
}

\author{
par Annick Alperovitch*
}

\begin{abstract}
L'application des concepts et des méthodes de la théorie de la décision conduit à une analyse rationnelle et quantitative de la démarche médicale, séduisante dans son principe, mais qu'il n'est pas toujours facile de mettre en œuvre. Aussi semble-t-il important que les spécialistes de la théorie de la décision insistent, plus qu'ils ne le font habituellement, sur le champ d'application et les limites de cette méthodologie dans le domaine médical. Dans cette présentation critique, il faut faire ressortir aussi les effets secondaires de ce type d'analyse du processus de décision qui, en médecine comme dans d'autres disciplines, s'avèrent souvent plus importants et plus intéressants que l'objectif principal. C'est dans cette perspective que je voudrais faire quelques remarques brèves sur l'introduction de la notion d'utilité dans les décisions médicales, l'analyse de la décision au lit du malade, la formation des cliniciens à la prise de décision.
\end{abstract}

\section{La notion d'utilité dans les décisions médicales}

Combien de médecins s'interrogent systématiquement au moment de prescrire un examen complémentaire sur les possibles conséquences de son résultat sur leur démarche ultérieure? Dans les essais thérapeutiques concernant des maladies mettant en jeu le pronostic vital, le critère privilégié est le taux de survie; mais ne faudrait-il pas tenir compte aussi, plus fréquemment, et plus nettement, de la qualité de la survie $(1,2)$ ? Les stratégies médicales, qu'elles concernent des problèmes diagnostiques et thérapeutiques, sont évaluées en fonction de critères, considérés comme objectifs, qui ne peuvent résumer l'ensemble des conséquences de la prescription du médecin, telles que peut les vivre le malade. C'est un apport essentiel de la thèorie de la décision que d'avoir introduit la notion d'utilité en médecine formalisant la valeur subjective que chacun peut attacher à un certain état de santé. Les médecins, en général, perçoivent très clairement le sens de cette notion, l'existence d'une grande variabilité interpatients et son influence potentielle sur la solution adoptée pour chacun.

\footnotetext{
* Docteur, Directeur de Recherches. Institut National de la Santé et de la Recherche Médicale, Villejuif, Cedex 94807 , France.
} 
Mais la pratique de cette méthodologie pose quelques questions essentielles. En effet, un ensemble d'hypothèses définit les conditions d'existence d'une fonction d'utilité (3). Parmi les médecins auteurs de travaux où intervient la notion d'utilité, peu soupçonnent l'existence de ces hypothèses et moins encore connaissent leur nature. Considérons la première de ces hypothèses: elle stipule que le sujet (ici le patient) analysant deux situations possibles $S_{1}$ et $S_{2}$, par exemple les conséquences sur son état de deux procédures thérapeutiques, est capable de dire s'il préfère $S_{1}$ à $S_{2}$, ou l'inverse, ou s'il les considère comme équivalentes. Pour que cette hypothèse soit vérifiée, il ne suffit pas, à mon avis, que le patient ait dit qu'il préfère, par exemple, $S_{1}$ à $S_{2}$ : chacun peut fournir une réponse sans pour autant avoir ressenti, compris, mesuré ce qu'étaient - ou plutôt ce que seraient - les situations $S_{1}$ et $S_{2}$. Dans un tel cas, la suite de la procédure, basée sur l'expression de cette préférence, perd tout son sens, quelles que soient la finesse de l'analyse et la rigueur du raisonnement. Lorsque le médecin décrit à un patient l'ensemble des résultats possibles, positifs et négatifs, parfois lointains, d'une option thérapeutique, comment savoir ce que le patient comprend de ce discours? Que peut signifier pour un individu qui n'est ni médecin, ni statisticien, une information sur la probabilité d'une séquelle ou le risque de rechute. Le patient, dans un contexte de stress, peut-il comparer, pondérer, agréger, les multiples critères à prendre en compte pour décider, ou plus exactement pour maitriser vraiment sa décision? Je doute, à titre personnel que cela soit souvent possible. Les recherches sur la psychologie de la décision permettent de progresser dans la compréhension de ces mécanismes mais ne peuvent vraisemblablement lever la difficulté fondamentale que je viens d'évoquer (4). Peut-être est-ce parce qu'il a conscience de ne pouvoir transmettre au malade toute l'information dont il dispose que le médecin, dans la majorité des cas, prend la décision; le malade s'exprime en l'acceptant ou la refusant (5).

L'utilité que l'on veut mesurer est influencée par de multiples critères. Dans une approche analytique, on peut tenter d'évaluer, critère par critère, l'utilité associée par le patient à chaque résultat possible des procédures que l'on veut comparer. A partir de ces fonctions unicritères, il faut alors définir une fonction d'utilité globale, multicritères. La plupart des études conduites selon ce protocole achoppent à ce stade. La définition mathématique de cette fonction nécessite des calculs dont il n'est pas sûr qu'ils aboutissent à un résultat. En effet, le type de cette fonction dépend des hypothèses sur les relations entre les fonctions d'utilité associées à chaque critère. L'expression de l'utilité multicritères à partir des fonctions élémentaires est d'autant plus simple que ces hypothèses sont fortes $(6)$. Lorsque la fonction d'utilité globale est établie à partir de règles définies a priori - et c'est le cas le plus fréquent - , on lui assigne une forme simple. Dans la majorité des travaux, on exprime l'utilité multicritères par une combinaison linéaire des utilités associées à chaque critère. Mais cette forme linéaire n'existe qu'en l'absence d'interaction entre les critères. Prenons un exemple du sens de cette absence d'interaction. Pour le critère douleur, l'utilité croît quand l'intensité de la douleur diminue. Pour le critère espérance de vie, pris isolément, l'utilité augmente lorsque cette espérance s'allonge. Mais cette relation positive entre l'espérance de vie et l'utilité peut être modifiée en fonction du critère douleur: si l'on doit souffrir beaucoup peut être préférera-t-on de ne pas devoir trop longtemps subir cette souffrance. On soupçonne une interaction aussi triviale; mais il en est d'autres plus complexes, plus subtiles, spécifiques à un individu donné, qui peuvent être complètement ignorées lorsque que l'on se base sur des estimations obtenues critère par critère.

Pour l'ensemble des raisons que je viens d'évoquer, il faut, je crois, que les cliniciens qui veulent développer une telle approche aient clairement à l'esprit ses limites. Il ne suffit pas 
d'avoir, à partir des réponses du malade, associé un nombre compris entre 0 et 1 à chacun des états possibles pour avoir défini une fonction d'utilité sur l'ensemble de ces états. Il faut alors rester prudent dans les conclusions que l'on peut tirer en manipulant ces nombres comme les valeurs d'une fonction d'utilité. Cette réserve ne s'inspire pas d'une orthodoxie de nature méthodologique, mais tient aux évidents dangers potentiels d'une telle erreur de jugement. Il n'existe pas toujours de cohérence dans nos préférences: forcer à la cohérence n'aboutira pas à prendre les décisions optimales. En outre, notre cohérence interne, lorsqu'elle existe, obéit souvent à des lois complexes qu'il faudrait beaucoup de temps pour expliciter.

\section{Les systèmes de décision au lit du malade}

Que penser alors des systèmes de décision au lit du malade, lorsque l'on est aussi circonspect sur l'un des aspects essentiels de la théorie de la décision: l'évaluation des utilités? La situation est différente dans la mesure oủ ces systèmes sont souvent orientés vers la prise de décisions plus techniques. L'exemple le plus courant est le choix d'une stratégie d'examens complémentaires, en vue de réduire l'incertitude diagnostique á un niveau permettant une décision thérapeutique. Les paramètres des modèles sont d'estimation plus facile: probabilités a priori des hypothèses de diagnostic, probabilités conditionnelles des résultats des examens complémentaires (permettant d'apprécier leur valeur discriminante), coûts, délais de réponse. De tels systèmes pourraient guider la démarche diagnostique si l'on dispose de plusieurs examens complémentaires que l'on souhaite ordonner de façon optimale pour aboutir au diagnostic au moindre coût. Les méthodes mises en œuvre se situent à l'intersection de la théorie de la décision et de la recherche opérationnelle. L'intérêt de tels systemes, dont la mise au point nécessite un travail multidisciplinaire important, est d'autant plus grand que le problème à résoudre est complexe et qu'aucune des stratégies possibles ne s'impose au terme de l'analyse du médecin. Si tel est le cas, il n'est guère vraisemblable que l'analyse du problème par l'intermédiaire d'un système d'aide à la décision détecte une stratégie qui surpasse très nettement les autres. Selon le problème, et pour un problème donné selon le profil diagnostique, il existe un sous-ensemble de stratégies dont les coûts estimés sont très voisins. Prenons un exemple qui n'est pas du domaine du diagnostic mais concerne les stratégies thérapeutiques. Les ménorragies (hémorragies menstruelles) importantes et répétées posent le problème de l'hystérectomie. Lorsque l'on évalue les conséquences d'une hystérectomie comparées à celles de l'absence de traitement chirurgical en terme d'espérance de vie, on trouve, pour une femme de 50 ans par exemple, une différence de 60 jours sur une espérance de vie de 29 ans, en faveur de la seconde solution (pas d'hystérectomie) (7). On pourrait discuter de l'utilisation du critère espérance de vie dans cette pathologie relativement bénigne, mais tel n'est pas mon propos ici. Cet exemple illustre un résultat général: les systèmes d'aide à la décision hésitent, en quelque sorte, lorsque les experts hésitent. Il faut montrer aux cliniciens qu'il ne peut en être autrement: il n'y a aucune formule magique dans les modèles d'aide à la décision. Il faut ensuite les persuader que i) si en tant qu'experts, ils ne savent vraiment pas quelle solution est la meilleure, ii) si la rationalité formalisée par le modèle leur paraît correspondre à leur propre conception, iii) si les estimations des différents paramètres sont les plus exactes dont on puisse disposer, alors il est raisonnable d'adopter la stratégie proposée en tête par le systeme. Et qu'en aucun cas, on ne peut tirer argument du faible écart entre cette stratégie et les suivantes pour conclure que le systėme est défaillant et rejeter en bloc les solutions proposées. 
Je n'ai jusqu'à présent envisagé que des systèmes devant traiter de problèmes relativement complexes auxquels peuvent être confrontés des cliniciens expérimentés. Certains espèrent beaucoup de systèmes dits experts (8) auxquels pourraient avoir accès de jeunes médecins, ou des généralistes devant faire face à des problèmes qu'ils ont rarement l'occasion de rencontrer. Il est encore trop tôt pour dresser un bilan, même préliminaire, de telles expériences qui restent d'une extrême rareté, bien que le premier système expert dans le domaine médical ait été conçu il y a une vingtaine d'années. Mais comme le soulignait récemment le Pr. Maurice Tubiana (9), aucun système expert ne peut transmettre toute la compétence de l'expert. Lorsque la conclusion diagnostique dépend de la présence ou de l'absence de tel signe clinique, il est à peu près sur que la sensibilité et la spécificité de ce signe, telles qu'elles résultent de l'observation clinique d'un médecin peu accoutumé à rechercher ce signe, sont différentes de celles estimées par l'expert. Dans le développement des systèmes experts, il faut donc avoir à l'esprit cette dimension fondamentale de l'expérience du clinicien et considérer avec prudence toute application dans laquelle le savoir de l'expert se manifeste non seulement dans le traitement des informations mais aussi dans le recueil de ces informations.

\section{Enseigner la théorie de la décision aux médecins}

Les médecins prennent des décisions. Autour de la prise de décision, s'est développé une théorie. Dans différents domaines, des exemples montrent l'intérêt de la théorie de la décision. Cependant, parmi les applications médicales de cette théorie très peu m'ont semblé convaincantes. Cette réserve tient, essentiellement, à la façon dont sont présentées ces applications, à travers les publications dans les revues médicales: les techniques d'aide à la décision servent à fabriquer un outil devant, schématiquement, améliorer la performance du médecin, à condition que celui-ci veuille bien s'en servir. Mais le meilleur outil de décision de l'homme c'est, probablement, son cerveau. Et il me semble plus efficace de vouloir améliorer cet outil de l'intérieur que de lui proposer une aide extérieure en cas de défaillance. C'est donc sur la place que doit avoir en médecine l'enseignement de la théorie de la décision, de ses principes, méthodes, et applications que je voudrais conclure ce commentaire.

J'ai participé à plusieurs reprises à la conception de systèmes d'aide à la décision, ayant nécessité une étroite collaboration entre méthodologistes et cliniciens (10-11). Au terme de chacune de ces expériences, le bilan des médecins fut identique: une certaine déception vis-à-vis de l'objectif principal, mais le sentiment d'avoir acquis quelque chose de fondamental. Leur façon de poser les problèmes de décision et de les résoudre était, de leur propre aveu, profondément modifiée par cette expérience et cette nouvelle approche s'étendait à l'ensemble de leur pratique.

Dans un travail sur l'aide au diagnostic des ictères, nous avions demandé à des médecins spécialistes de classer, selon leur gravité, différentes erreurs possibles de diagnostic (12). Si d'une façon générale, il existait une bonne concordance entre les classements, on pouvait noter cependant quelques divergences marquées, un médecin situant à une extrémité de l'échelle de gravité telle erreur que ses collègues mettaient plutôt à l'extrémité opposée. L'intérêt de mettre en lumière ces divergences est évident; et les discussions qu'elles entrấnent témoignent de l'importance de telles observations. L'appréciation subjective de la gravité d'une erreur de diagnostic conditionne, en effet, l'ensemble de la démarche du médecin, de la prescription des examens complémentaires à la recherche du diagnostic, comme la 
décision thérapeutique. Cette étude de la gravité des erreurs de diagnostic, permettant d'appréhender la notion de regret telle quelle est définie dans la théorie de la décision, a donc révélé, d'une façon très frappante des différences de vue qui ne s'étaient pas exprimées jusqu'alors. Au-delà des discussions à la recherche d'un consensus, ces divergences peuvent par exemple conduire à la réalisation d'essais thérapeutiques lorsque, comme c'était le cas dans cette étude, elles tiennent à des différences dans l'évaluation de l'efficacité des thérapeutiques. En effet, un médecin jugera une erreur de diagnostic d'autant moins grave que le traitement de la maladie réelle est moins efficace.

La formation des médecins à la prise de décision par un enseignement de la théorie de la décision ne doit pas intervenir trop tôt dans les études médicales. Pour des étudiants n'ayant aucune pratique, il ne peut s'agir que d'un exercice académique. Il faut avoir été confronté aux difficultés de la décision médicale pour réellement comprendre la portée de cet enseignement. Je ne suis pas sûre, d'ailleurs qu'il soit vraiment utile d'essayer de convaincre les médecins de la puissance de ce type de raisonnement, en leur montrant les retombées pratiques qu'a pu avoir, ici ou là, une telle approche. Il me semble que la théorie de la décision et les concepts qui s'y rattachent ont, en eux-mêmes, une force capable d'influencer le raisonnement et de modifier durablement le processus de la décision médicale.

\section{RÉFÉRENCES}

1. SPITZER, W.O., DOBSON, A.J., HALL, J., CHESTERMAN, E., LEVI, J., SHEPHERD, R., BATTISTA, R., CATCHLOVE, B.R.: "Measuring the quality of life of cancer patients. A concese QL-index for use by physicians". J. Chron. Dis., 1981, 34: 585-598.

2. PAUKER, S.G., McNEIL, B.J.: "Impact of patient preferences on the selection of therapy".J.Chron. Dis., 1981, 34: 77-86.

3. CHERNOFF, H., MOSES, L.E.: "Elementary decision theory". John Wiley and Sons, Inc., 1963.

4. KAHNEMAN, D., EVERSKY, A.: "The psychology of preferences". Scient. Amer, 1982, 246: $136-142$.

5. CARD, W.I., GOOD, I.J.: "The estimation of implicit utilities of medical consultants". Math. Biosciences, 1970, 6: 45-54.

6. KEENEY, R.L., RAIFFA, H.: "Decisions with multiple objectives". John Wiley and Sons, Inc., 1976.

7. BUNKER, J.P., Mc PHERSON, K., HENNEMAN, P.L.: "Elective hysterectomy. Dans: Costs, risks and benefits of surgery". Bunker, J.P., Barnes, B.A., Mosteller, F., Eds. Oxford univ. Press, 1977.

8. MILLER, R.A., POPLE, H.E., MYERS, J.D.: "Internist-I, an experimental computer-based diagnostic consultant for general internal medicine". N. Engl. J. Med., 1982, 307: 468-476.

9. TUBIANA, M. Intervention au colloque: "Jugement et décision en cancérologie". Bordeaux, Avril 1986.

10. FRAGU, P., ALPÉROVITCH, A., PATOIS, E.: "Screening and diagnosis of hyperthyroÏdism: an attemptat test reduction”. J. Epid. Com. Health, 1979, 33: 215-218.

11. SAINT-MARC GIRARDIN, M.F., LE MINOR, M., ALPÉROVITCH, A., ROUDOT-THORAVAL, F., METREAU, J.M., DHUMEAUX, D.: "Computer-aided selection of diagnostic tests in jaundiced patients". Gut, 1985, 26: 961-967.

12. LE MINOR, M., ALPÉROVITCH, A., KNILL-JONES, R.P.: "Applying decision the ory to medical decision making-concept of regret and error of diagnosis". Meth. Inf. Med., 1982, 21: 3-8. 